\title{
Interference between mnemonic and categorical organization in memory
}

\author{
B. GOVARDHAN REDDY \\ Osmania University, Hyderabad, India \\ and \\ FRANCIS S. BELLEZZA \\ Ohio University, Athens, Ohio
}

\begin{abstract}
The purpose of the experiment reported here was to determine whether a mnemonic device could be used as an auxiliary means of memorizing category words and thereby optimize recall performance. The answer is clearly "no." When subjects tried to use the pegword mnemonic to memorize category words, recall was poorer than when the category words were studied using no special mnemonic procedure. Retrospective reports by the subjects indicated that the obvious categorical relations among the words interfered with the implementation of the mnemonic device. It is concluded that a learner cannot use two schemas or strategies simultaneously to organize and, thus, remember a presented set of information.
\end{abstract}

Many students of memory have come to believe G. Mandler's (1967) dictum that to organize is to memorize and to memorize is to organize. Hence, a fruitful way to study memory has been to study the strategies and memory structures that people use to organize new information. One of the earliest investigations of the role of organization in learning involved having subjects learn a list of categorized words (Bousfield, 1953). Bousfield found that experimental subjects tend to recall together words from the same category, even if the words are not presented together in the list. This occurs because each word is recognized during study as a member of one of the small number of categories making up the list. As a consequence, the word becomes associated with its category label. During recall, the learner can then recall the category label and use it as a recall cue (Bower, Clark, Lesgold, \& Winzenz, 1969).

It has been discovered recently that category organization does not result in the optimal recall of category words. Rabinowitz and J. Mandler (1983) presented subjects with materials that could be organized either into categories, such as food or clothing, or into schematic activities, such as skiing or going to a ballet. Furthermore, in one of their experiments, they demonstrated that category organization was more salient than schematic organization. Nevertheless, schematic organization resulted in better recall than did categorical organization.

In contrast to Rabinowitz and J. Mandler's (1983) results, it is possible that organizational structures other

This experiment was part of a thesis written by the first author under the direction of the second author in partial fulfillment of the requirements for the PhD at Ohio University.

Requests for reprints may be sent to F. S. Bellezza, Department of Psychology, Ohio University, Athens, OH 45701. than schemas may enhance the recall of category words. To be specific, research on mnemonic devices has demonstrated that organizational strategies utilized by the learner can result in high levels of recall performance. Recall is enhanced even when the nature of the organization may not be obvious from inspection of the material presented (Bellezza, 1981). An example of this is the story mnemonic (Bower \& Clark, 1969), in which a list of randomly selected nouns can be accurately recalled if the learner first fabricates a story that incorporates the nouns in the list.

Bellezza (1983) argued that a mnemonic device can function as a supplementary form of organization, even under conditions in which the information to be remembered is recognized as having some inherent organization other than that created by the mnemonic device. That is, when information is presented that the learner recognizes as belonging to some obvious category or as being part of some schema, the learner may use a mnemonic device along with the salient category or schema to further organize and retain the information in memory.

The purpose of the experiment discussed here was to determine whether the recall of category words could be enhanced by using a mnemonic device to further organize them.

\section{METHOD}

\section{Subjects}

Seventy-two undergraduates enrolled in introductory psychology courses at Ohio University volunteered to participate for extra course credit.

\footnotetext{
Materials

Two lists consisting of six nonexhaustive categories of 7 words each were sampled from the Battig and Montague (1969) category norms. The categories selected represented concrete objects, and care was taken
} 
to minimize any intercategory associations. Also, two lists of 42 random nouns were selected from the Paivio, Yuille, and Madigan (1968) norms. Each word selected had an imagery rating between 6.01 and 7.00. In addition, 42 nouns were chosen to be used as mnemonic pegwords. These also had imagery ratings between 6.01 and 7.00.

\section{Procedure}

Twelve subjects were randomly assigned to each of the six experimental conditions. The six conditions were formed by crossing the factor of word condition (category words vs. random words) with the strategy condition (free recall, link mnemonic, and pegword mnemonic). Subjects were tested individually, and each list word was presented visually on an index card by the experimenter at the rate of one word every $6 \mathrm{sec}$. Subjects in the category-words condition were presented one of the two lists of category words in which all the words in the same category were adjacent in the list. Subjects in the random-words condition were presented one of the two lists of random words.

Subjects using either the link-mnemonic or the pegword-mnemonic strategy were instructed in the use of these mnemonic devices. Subjects in the link-mnemonic condition were instructed to form a visual image of each noun when it appeared and to associate it to the image of the previous noun in the list. Hence, subjects were to use visual imagery to link the 41 pairs formed by the 42 nouns (Bellezza, 1982, chap. 2). Subjects in the pegword-mnemonic condition were presented a list of 42 pegwords on a card which was always available to them. They did not have to memorize the pegwords. As each list word was presented, the subject had to form an interacting image consisting of the list word and that pegword in the same corresponding position in the pegword list. That is, the subject had to associate the first list word with the first pegword, the second list word with the second pegword, and so on. Subjects in the free-recall condition were not given any special learning instructions.

After all the words had been presented, the subjects engaged in a distractor task for $2 \mathrm{~min}$. Subjects in the free-recall condition were asked to recall the words in any order. Subjects in the link-mnemonic condition were given the first word from the list and asked to use their visual images to recall the rest of the words in the list. Subjects in the pegwordmnemonic condition had the pegwords available to them. They were instructed to form a visual image of each pegword and to use this image to recall the list word paired with it.

\section{RESULTS}

The proportion of words recalled in each of the six experimental conditions is shown in Figure 1. Strategy condition was marginally significant $[F(2,66)=3.41$, $M S e=.224, p<.05]$. Word condition was not significant $(F<1)$. The most important result was the significant strategy condition $\times$ word condition interaction $[F(2,66)=26.46, M S e=.224, p<.001]$. As can be seen in Figure 1, when random words were presented, the pegword mnemonic resulted in the best recall. This result is not surprising (Battig \& Bellezza, 1979). When categorized words were presented, however, the freerecall instructions resulted in the best recall performance. This result is surprising. Bellezza (1983) proposed that a mnemonic strategy can be used as a supplementary organizational strategy to improve recall performance in those situations in which the organizational structure activated is known not to result in optimal recall performance. This hypothesis appears to be incorrect.

\section{DISCUSSION}

In the free-recall condition, in which subjects were not instructed in a mnemonic technique, .80 of the category words were recalled, but

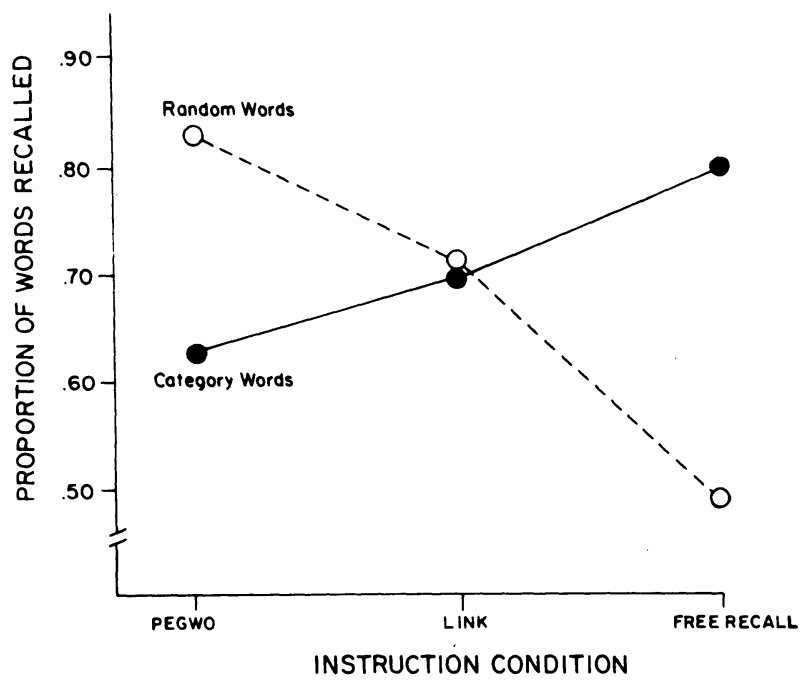

Figure 1. Proportion of category and random words recalled for the free-recall, link-mnemonic, and pegword-mnemonic conditions.

only .49 of the random words. The categories were salient and utilized by the subjects studying the categorized lists. Because words from the same category were presented together, they tended to be recalled together.

When the pegword mnemonic was used, .83 of the random words were recalled. This high level of recall performance demonstrates the effectiveness of the pegword mnemonic. The important result, however, was the poor recall of the category words when using the pegword mnemonic. The proportion of category words recalled was .63 . What is the explanation for this? Informal questioning after the experiment revealed that each subject in this condition soon became aware of the categorized nature of the list, and this interfered with his or her strategy of using the pegword mnemonic. In other words, the categorical relations apparent in the structure of the list attracted the subject's attention. Because of this, the necessary composite images incorporating one list word and one pegword could not be formed. In a reciprocal fashion, the attempt to use the pegword mnemonic interfered with the subjects' attending fully to the categorical organization. Because of this mutual interference, attempting to use the pegword mnemonic on categorized word lists resulted in recall that was worse than recall resulting from simply studying a categorized list or using the pegword mnemonic on a random list. Further research is required to determine whether interference between the two types of organization occurred not only during study but also during recall.

Using the link mnemonic to recall the random words was not as effective as was using the pegword mnemonic. This result is not surprising, because when subjects use the link mnemonic, recall of each word is dependent on the successful recall of the previous word in the list. Failure to recall any word in the list reduces the probability of recalling any of the following words (Battig \& Bellezza, 1979). Recall of the category words, however, was better when subjects used the link mnemonic than when they used the pegword mnemonic. The categorical nature of the words may not have interfered with the link mnemonic as much as it interfered with the pegword mnemonic. In the link mnemonic, the words are linked together directly. It appears that this can be done with words from the same category.

Bellezza (1983) suggested that a mnemonic device can be used as an auxiliary strategy for further organizing and memorizing material. From this point of view, the mnemonic organization should supplement the categorical or schematic organization automatically activated by the semantic content of the material. Our results indicate that this type of additional processing seems to be impossible. It appears that subjects cannot simultaneously use two methods of organization to remember information. Another possible strategy might be to have subjects study the material twice, using a different method of organization each time, 
and to recall the material twice, again using the appropriate method of organization each time. Other research, however, indicates that this procedure also does not result in optimal recall performance. The results of Young and Bellezza (1982) indicate that if material is studied on more than one occasion, then recall performance is better if the same method of organizing the material is used each time the material is presented.

\section{REFERENCES}

Battig, W. F., \& Bellezza, F. S. (1979). Organization and levels of processing. In C. R. Puff (Ed.), Memory organization and structure (pp. 321-346). New York: Academic Press.

Battig, W. F., \& Montague, W. E. (1969). Category norms for verbal items in 56 categories: A replication and extension of the Connecticut category norms. Journal of Experimental Psychology Monograph, 80(3, Pt. 2).

BellezzA, F. S. (1981). Mnemonic devices: Classification, characteristics, and criteria. Review of Educational Research, 51, 247-275.

BELLEZZA, F. S. (1982). Improve your memory skills. Englewood Cliffs, NJ: Prentice-Hall.

BellezZA, F. S. (1983). Mnemonic-device instruction with adults. In M. Pressley \& J. R. Levin (Eds.), Cognitive strategy research: Psychological foundations. New York: Springer-Verlag.
Bousfield, W. A. (1953). The occurrence of clustering in the recall of randomly arranged associates. The Journal of General Psychology, 49, 229-240

Bower, G. H., \& CLARK, M. C. (1969). Narrative stories as mediators for serial learning. Psychonomic Science, 14, 181-182.

Bower, G. H., Clark, M. C., Lesgold, A. M., \& Winzenz, D. (1969). Hierarchical retrieval schemes in recall of categorized word lists. Journal of Verbal Learning \& Verbal Behavior, 8, 323-343.

MANDler, G. (1967). Organization and memory. In K. W. Spence \& J. T. Spence (Eds.), The Psychology of Learning and Motivation (Vol. 1, pp. 327-372). New York: Academic Press.

Paivio, A., Yuille, J. C., \& Madigan, S. A. (1968). Concreteness, imagery, and meaningfulness values for 925 nouns. Journal of Experimental Psychology Monograph, 76(1, Pt. 2).

Rabinowitz, M., \& MANDLER, J. M. (1983). Organization and information retrieval. Journal of Experimental Psychology: Learning, Memory, \& Cognition, 9, 430-439.

Young, D. R., \& BellezzA, F. S. (1982). Encoding variability, memory organization, and the repetition effect. Journal of Experimental Psychology: Learning, Memory, \& Cognition, 8, 545-559.

(Manuscript received for publication February 13, 1986.) 